\title{
ASYMMETRY AND PERIODICITIES IN SOLAR ACTIVITY
}

\author{
M. CARBONELL, R. OLIVER AND J.L. BALLESTER \\ Departament de Fisica. Universitat de les Hles Balears. E-07071 Palma \\ de Mallorca. Spain
}

ABSTRACT The historical record of daily sunspot areas (1874-1989) has been analysed, looking for the short (155 days) and intermediate (323 and 540 days) term periodicities. Also the North - South asymmetry during those years has been studied.

\section{INTRODUCTION}

During recent years the presence of short and intermediate term periodicities in several features of solar activity has been reported.

A 155-day periodicity has been detected in the solar flare occurrence rate, the sunspot area variations, the Zurich sunspot number and the aurorae record.

A 323-day periodicity has been reported in the Zurich sunspot number, in the solar diameter measurements, in the sunspot blocking function, in the 10.7 $\mathrm{cm}$ radio flux, and the CaII $\mathrm{K}$ plage index.

The 540-day periodicity has been found in the $10.7 \mathrm{~cm}$ radio flux, in solar flares, in sunspot areas during solar cycle 21 and the observations of the solar horizontal diameter.

On the other hand, the existence of a North-South asymmetry in solar activity is generally accepted, and it has been detected in most of its manifestations (solar flares, sunspots, magnetic flux, etc.) although a detailed study of its behaviour is still missing.

Sunspots are key features of solar activity, being a measure of the amount of magnetic flux emerging through the photosphere, and they have been one of the main indices of solar activity for a long time. For this reason, we have chosen the historical record (1878-1989) of daily sunspot areas to assess the presence of those periodicities and to study the behaviour of its North - South asymmetry.

\section{DATA AND TECHNIQUES}

The Greenwich Photoheliographic Results (1874-1982) and the Unites States Air Force (1983-1989) have provided us with the total daily area of visible sunspots expressed in millionths of the Sun's visible hemisphere. To look for the periodicities we have adopted the Lomb-Scargle Periodogram (Lomb, 1976; Scargle 1982), modified by Horne \& Baliunas (1986), which is appropiate for the analysis of unevenly-spaced data. Scargle (1982) and Horne \& Baliunas (1986) have provided with a convenient estimate (the false alarm probability, FAP) for the 
probability that a given peak be a true signal. Moreover, we have set our FAP at a height that implies a very restrictive condition for the peak's significance, i.e. the probability that a peak that exceeds the FAP level due to chance is smaller than $0.01 \%$. The periodogram has been computed at 400 equally spaced frequencies over the frequency interval $57-115 \mathrm{nHz}$ (100-200 days) and $19-39 \mathrm{nHz}$ (300-600 days). This number of frequencies is sufficient to obtain the resolution of all the peaks present in the portion of the periodogram under study.

To analyse the behaviour of the asymmetry, we have constructed an asymmetry time series and studied it by means of the following methods: 1) Power spectrum and statistical analysis. 2) Montecarlo simulations. 3) Chaotic analysis. 4) Multifractal analysis.

\section{RESULTS}

The picture we have obtained from our study of daily sunspot areas is that a short periodicity between 150-160 days seems to be present, in a significant way, from cycle 16 to 21 . The period changes from one cycle to the next when whole individual cycles are considered. However, during cycles 12, 14, 16, 17, 18, 19, 20,21 , a significant peak at 155.6 days is found in some time intervals, this being a feature suggesting intermittency. Also, a non coherence in the phase is found during solar cycles 16-18 which could be due to different times in the trigger of the periodicity suggesting, again, intermittency. If this short periodicity is a true feature of solar activity, it would imply that the emergence of magnetic flux presents a periodicity, perhaps intermittent. Thus, the periodic emergence of magnetic flux could trigger, via magnetic reconnection with the old flux, flares, giving place to the periodicity found in them.

Concerning the periodicity at 323 days, the results indicate that it is only confirmed for solar cycle 21 while its presence throughout the historical record of solar activity is very doubtful.

The situation is different for the periodicity around 540 days. Its presence is well established for cycle 19 and appears clearly in cycle 18. Also, during cycles 12,14 and 17 there are indications of its presence.

The study of the North-South asymmetry in sunspot areas has produced the following results : 1) The asymmetry is a real feature in the North-South distribution of sunspot areas. 2) The asymmetry time series can be modelled by means of a multicomponent model made by a linear trend, a deterministic cycle with a period around 12.5 years and a dominant purely random component which accounts for most of the variance of the time series. 3) Statistical rank correlation tests confirm the existence of an anticorrelation between the asymmetry and the solar cycle. 4) Montecarlo simulations indicate that the real asymmetry cannot be obtained randomly from a binomial or uniform distribution of probability between hemispheres. 5) Chaotic analysis reveals that the asymmetry time series has a stochastic behaviour. 6) The multifractal properties of the asymmetry time series indicate that it presents temporal intermittency.

A more extense information about this research can be found in Carbonell \& Ballester, (1990); Carbonell \& Ballester, (1991); Oliver et al., (1992); Carbonell \& Ballester, (1992) and Carbonell et al., (1992). 


\section{ACKNOWLEDGMENTS}

We would like to acknowledge financial support from DGICYT under grant PS89-0148.

\section{REFERENCES}

Carbonell, M., Ballester, J.L., 1990, A\&A 238, 377.

Carbonell, M., Ballester, J.L., 1991, A\&A 249, 295.

Carbonell, M., Ballester, J.L., 1992, A\&A 255, 350.

Carbonell, M., Oliver, R., Ballester, J.L., 1992 (Submitted).

Horne, J.H., Baliunas, S.L., 1986, ApJ 302, 757.

Lomb, N., 1976, Ap\&SS 39, 447.

Oliver, R., Carbonell, M., Ballester, J.L., 1992, Sol. Phys. 137, 141.

Scargle, J.D., 1982, ApJ 263, 835. 